\title{
Numerical Simulation of Swirling Flows - Heat Transfer Enhancement
}

\author{
Teresa Parra ${ }^{1}$, Ruben Perez ${ }^{1}$, Miguel A. Rodriguez ${ }^{1}$, Francisco Castro1, \\ Robert Z. Szasz ${ }^{2}$, Artur Gutkowski ${ }^{3}$ \\ 1University of Valladolid, Department of Energy and Fluid Mechanics \\ Paseo del Cauce 59, 47011 Valladolid, Spain \\ terpar@eii.uva.es; jrubenpd@gmail.com; miguel@eii.uva.es; castro@eii.uva.es \\ 2Lund University, Energy Division, Lund, Sweden \\ Robert-Zoltan.Szasz@energy.lth.se \\ ${ }^{3} \mathrm{Lodz}$ University of Technology, Institute of Turbomachinery \\ 219/223 Wolczańska, 90-924 Łódz, Poland \\ artur.gutkowski@p.lodz.pl
}

\begin{abstract}
In this paper, the influence of swirl number on mixing of two confined coaxial jets is analysed. The swirl generator is a set of fixed vanes located in the annular nozzle. Different trailing edge angles result in swirl numbers ranging from 0.14 to 0.95. Numerical model is validated using non reactive benchmarks. Low, intermediate and large swirling injectors are simulated to contrast the flow pattern. The presence of Inner Recirculation Zone associated to intermediate and high swirl numbers plays an important role in the heat and mass transfer since promote high gradients with minimum head loss. Low swirl injectors show large mixing regions in absence of the central vortex structure.
\end{abstract}

Keywords: Swirl number, CFD, mixing, recirculation zone.

(C) Copyright 2015 Authors - This is an Open Access article published under the Creative Commons Attribution License terms (http://creativecommons.org/licenses/by/3.0). Unrestricted use, distribution, and reproduction in any medium are permitted, provided the original work is properly cited.

\section{Introduction}

Swirling flows in burners are common because of the stabilization of lean flames with minimum head losses, ultra low emissions and fuel saving, [1-2]. This issue has important environment implications since the fuel slip due to incomplete combustion is a harmful greenhouse gas.

It is commonly known the mixing capacity is a feature of turbulent flows. It has been widely studied the heat transfer enhancement associated to the swirling flows. Guo et al. [3] carried out a theoretical study about the effect of a secondary flow on the laminar convective heat transfer. Saqr and Wahid [4] proposed an empirical correlation for predicting the entropy augmentation as a function of the swirl number.

Bearing in mind the quantification of thr enhanced heat transfer, Gul and Evin [5] showed that an increase of $300 \%$ of heat transfer can be achieved by placing a helical tape at the begining of the test section. Nuntadusit et al. [6] studied the effect on Nusselt number of different set ups of multiple swirling jets.

As for design of the swirl generators, Zohir et al. [7-8] evaluated the increase of heat transfer rate when modifying the location and pitch angle of the swirl generator upwind and downwind the test chamber.

\section{Physical Model}

The geometry of the burner is composed by two coaxial nozzles, a swirl generator (a number of fixed vanes on the annular nozzle) and the combustion chamber. Configuration corresponds to the experimental characterization performed by Roback and Johnson [9] being the only difference the swirl injector, such as is depicted in figure 1.

Despite the simple geometrical set-up of the benchmark, the flow pattern shows complex aerodynamic behaviour. The case considers two coaxial 
jets: one axial and another annular swirling jet. An expansion ratio of 4 in area produces the Outer Recirculation Zone (ORZ) when annular jet enters in the chamber. This kind of configuration has been analyzed by Palm [10] for swirl numbers $0,0.6$ and 1.2 .

Main difference in flow pattern between low and large swirling injectors is the presence of an expansion or a recirculation in the centre of the chamber. If swirl number is over 0.6 , the flow turns back into the centre and then vortex break down phenomenon appears to form an Inner Recirculation Zone (IRZ), [11]. Both recirculation zones are responsible of high shear and mixture. If swirl number is lower, there is an Inner Divergence Zone instead of the IRZ.

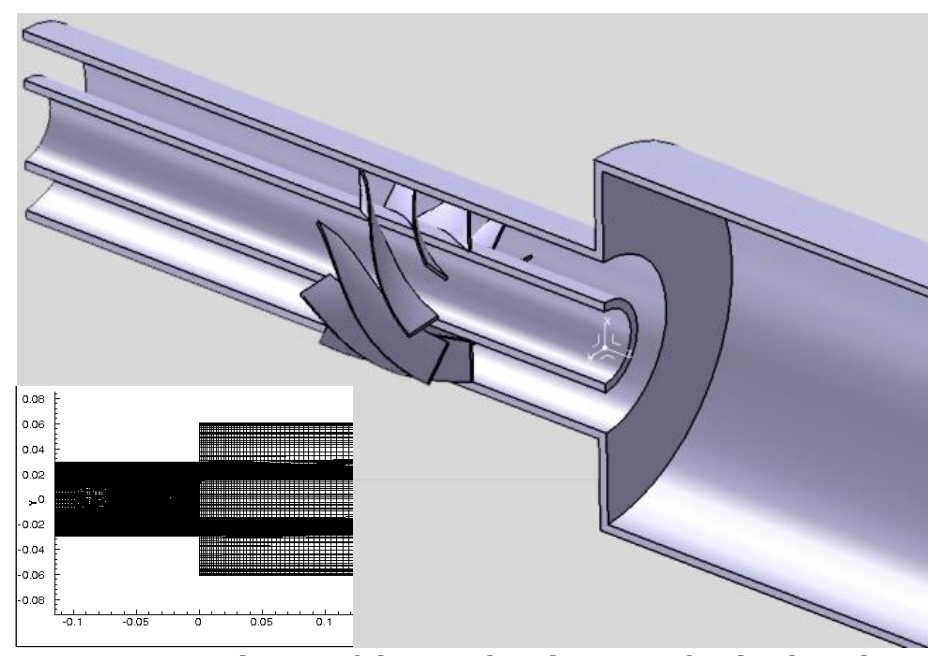

Figure 1. Scheme of the swirling burner of Roback and Johnson [9] with swirler angle 64을 ${ }^{\circ}$ and detail of the hexahedral mesh.

Computational Fluid Dynamics (CFD) has become a useful tool to gain insight of the phenomena involved in thermal and fluid mechanics processes. The simulation requests the generation of a mesh that is a discretization of a continuous geometry into a computational domain. The finite volume Ansys-Fluent was used to carry out the simulations.

The selection of numerical methods is a prerequisite to perform accurate numerical simulations to better predict the performance of the swirling flow burner.

\section{Numerical Model}

The conservative equations for 3D, steady, turbulent and incompressible flow are solved with a second order scheme looking for better accuracy. Table 1 summarizes the boundary conditions for the nozzle inlets working with air. Passive scalar let identify the mixture fraction associated to the inner jet. As for length scale the hydraulic diameter was considered. Main uncertainty is the transitional flow regime. The 3D mesh has 1.5 million hexahedral cells. The sensibility analysis of mesh resolution revealed that RANS models provide velocity profiles that were independent from meshes for more than 1.5 million cells. Meshes with 0.5, $0.751,1.5$ and 2 million cells were tested, as described in [12].

PISO was the algorithm for pressure-velocity coupling since the option provides faster convergence. Multigrid options provide a substantial reduction of the computational time. It consists on solving equations on a coarse mesh to get initial solution to iterate on a finer mesh. However, multigrid is not suitable for reactive cases because the averaging on temperature field is a precursor of the lack of accuracy on the reaction rate. Parallel processing is important to afford resolution of meshes with adequate spatial resolution. The domain decomposition process was established to minimize the processor boundaries and therefore, saving information transfer time.

Table 1. Boundary conditions for nozzles from reference [9].

\begin{tabular}{|l|l|l|}
\hline & Annular inlet & Central inlet \\
\hline $\begin{array}{l}\text { Radio } \\
\text { (inner-outer) (m) }\end{array}$ & $0.0128-$ & $0-0.0125$ \\
0.0295 & \\
\hline Velocity (m/s) & 1.54 & 0.66 \\
\hline Temperature (K) & 900 & 300 \\
\hline Turbulent Intensity (\%) & 7.5 & 12 \\
\hline Length scale (m) & 0.017 & 0.025 \\
\hline Passive scalar & 0 & 1 \\
\hline
\end{tabular}

\section{Turbulence Model}

As for the turbulence model, among RANS models of 1,2 or 4 equations, the most accurate was the $\mathrm{k}-\varepsilon$ RNG model dominated by the swirl. It is recommended by Ferziger [13-14] to deal with the non-isotropic turbulence associated with the swirling.

The conservation equations for the $\mathrm{k}$ and $\varepsilon$ are represented in the RNG model as:

$$
\frac{\partial}{\partial x_{i}}\left(\rho k u_{i}\right)=\frac{\partial}{\partial x_{j}}\left(\alpha_{k} \mu_{e f f} \frac{\partial k}{\partial x_{j}}\right)+G_{k}-\rho \varepsilon
$$

$$
\begin{aligned}
& \frac{\partial}{\partial x_{i}}\left(\rho \varepsilon u_{i}\right)=\frac{\partial}{\partial x_{j}}\left(\alpha_{\varepsilon} \mu_{e f f} \frac{\partial \varepsilon}{\partial x_{j}}\right) \\
& +C_{1 \varepsilon} \frac{\varepsilon}{k} G_{k}-C_{2 \varepsilon} \rho \frac{\varepsilon^{2}}{k}-R_{\varepsilon}
\end{aligned}
$$


being

$R_{\varepsilon}=\frac{C_{\mu} \eta^{3} \rho\left(1-\eta / \eta_{0}\right)}{1+\beta \eta^{3}} \frac{\varepsilon^{2}}{k}$

and

$\eta=\left(2 S_{i j} S_{i j}\right)^{0.5} k / \varepsilon$

Where $\mathrm{k}$ is the turbulent kinetic energy and $\varepsilon$ its corresponding dissipation, $\mathrm{u}$ denotes the averaged velocities and $\rho$ the density.

The source term $R_{\varepsilon}$ in equation 2 , represents the rate of strain defined in equations (3) and (4). Where $S$ is the strain tensor calculated based on averaged velocities.

The coefficients are summarized in table 2.

Table 2. Constants in the RNG k- $\varepsilon$ model.

\begin{tabular}{|c|c|c|c|c|}
\hline $\mathrm{C}_{\mu}$ & $\mathrm{C}_{1 \varepsilon}$ & $\mathrm{C}_{2 \varepsilon}$ & $\eta_{0}$ & $\beta$ \\
\hline 0.0845 & 1.42 & 1.68 & 4.38 & 0.012 \\
\hline
\end{tabular}

Bearing in mind the flow pattern is dominated by recirculation zones and fluid separation of the boundaries, the turbulence wall treatment was that of non equilibrium, hence it is not necessary to control the $y+$ range of variation.

\section{Swirl Generator}

Swirl generator is characterized by eight- fixed vanes in the annular nozzle with a fix angle in the trailing edge, Parra et al. [15]. The definition assumed for the swirl number is the relation between the flowaveraged-azimuthal velocity and the corresponding axial velocity, see equation 5 .

Table 3. Details of different set up of swirl injectors.

\begin{tabular}{|l|l|l|l|}
\hline Design & Flat plate & Curved blade & Flat plate \\
\hline Chord & $50 \mathrm{~mm}$ & $50 \mathrm{~mm}$ & $25 \mathrm{~mm}$ \\
\hline Swirler Angle & $22^{\mathrm{o}}$ & $54^{\mathrm{o}}$ & $64^{\mathbf{o}}$ \\
\hline Swirl number & 0.14 & 0.74 & 0.95 \\
\hline
\end{tabular}

$S=\frac{\int \rho r v_{\theta} v_{z} 2 \pi r d r}{R \int \rho v_{z}^{2} 2 \pi r d r}$

Hence different swirl numbers request different orientation for the vanes. Previous studies have verified the lack of influence of the swirler's chord on the swirl number as well as the weak influence of the design (flat plates or curved blades) on the final flow pattern. Table 3 shows the different swirler configurations presented in this paper to provide different swirl numbers.

\section{Influence of Swirl Number}

This section is devoted to the analysis of the flow pattern associated to low and high swirl numbers.

Figure 2 shows, for non-reactive case and swirl numbers of $0.14,0.74$ and 0.95 , the contours of temperature in a longitudinal plane and the volumes of iso-value null for axial velocity. Annular jet has a temperature of $900 \mathrm{~K}$ whereas central jet has $300 \mathrm{~K}$; hence temperature gradient suggests the mixing.

Besides, the zero axial velocity surfaces identify the position, shape and size of recirculation zones. Bearing in mind the ORZ that is formed due to the sudden expansion in the discharge of the annular nozzle [16], it has a diminishing in size while increasing the swirl number. This statement agrees with other research works [17].

Swirl number 0.74 shows an incipient IRZ while Swirl number 0.95 shows a larger IRZ. The IRZ forces the mixing; hence temperature is homogenized very close to the nozzle's discharge. This means that temperature is homogenized in more remote sections of the nozzle's discharge in the cases with low azimuthal velocity.

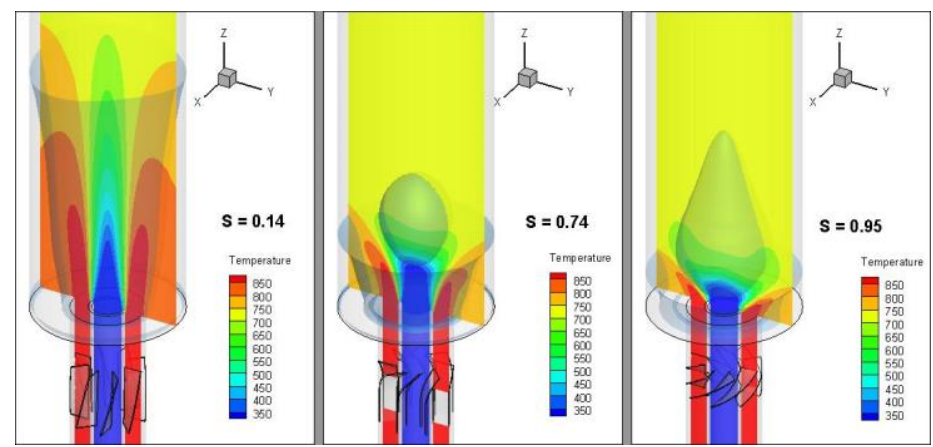

Figure 2. Contours of temperature in the plane $\mathrm{x}=0$ and isosurfaces of null axial velocity to locate recirculation zones. Left) Swirl no. 0.14, Center) Swirl no. 0.74 and Right) Swirl no. 0.95 .

Furthermore, the shape of the IRZ in the upstream stagnation point is convex for swirl number 0.74 and concave for swirl number 0.95 increasing significant effect on heat transfer for larger swirls such it was showed in [18]. 
IRZ plays an essential role in homogenizing temperature. Because of the IRZ, the shear layer is deflected. The bigger the IRZ is, the thinner the shear layer is. Hence higher gradient appears. Another aspect is that the upwind stagnation point of the IRZ is approaching the discharge of the central nozzle while increasing the swirl number; hence the mixing region is small.

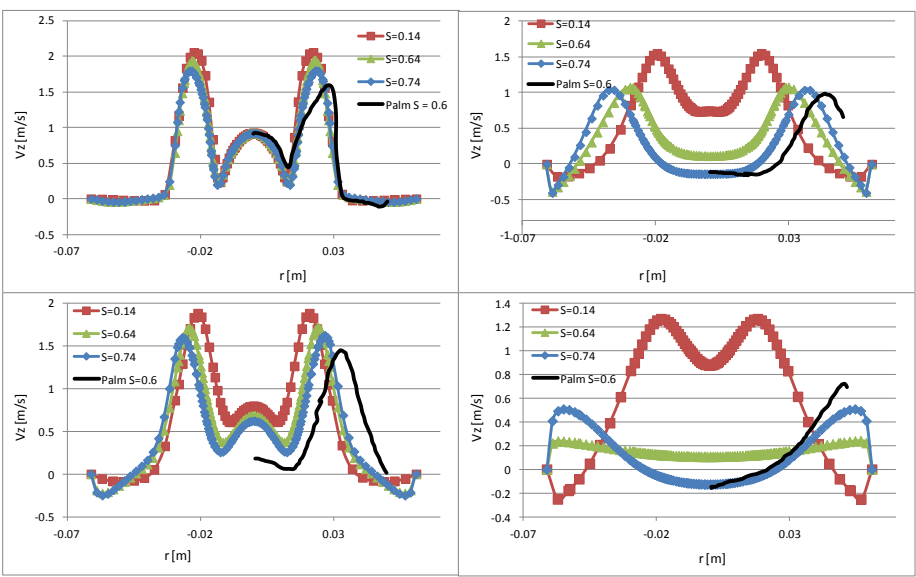

Figure 3. Radial profiles of axial velocities for different sections. Upper Left) $Z=5 \mathrm{~mm}$, Lower Left) $Z=25 \mathrm{~mm}$, Upper Right) $\mathrm{Z}=50 \mathrm{~mm}$ and Lower Right) $\mathrm{Z}=100 \mathrm{~mm}$ Experimental values of Palm et al. [10].

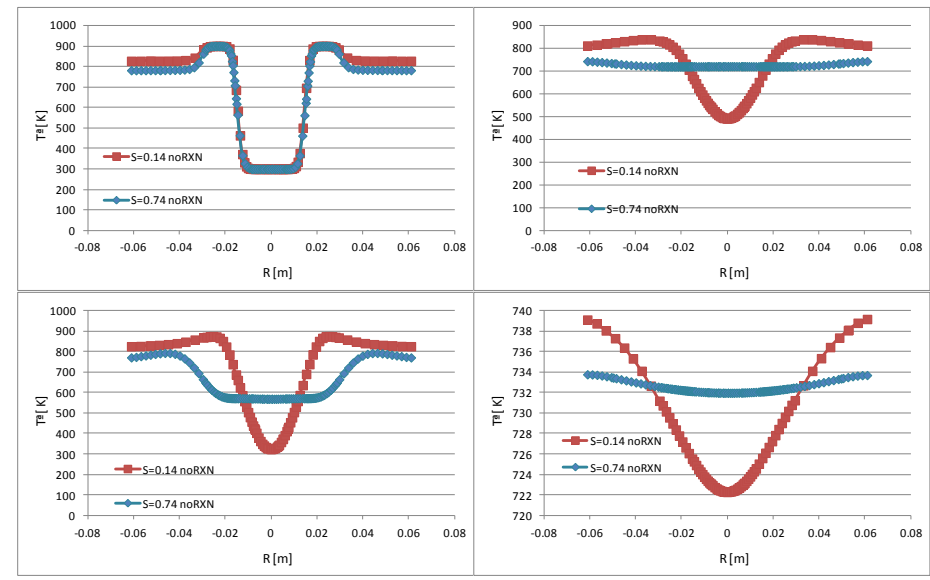

Figure 4. Radial profiles of Temperature for different sections. Upper Left) $Z=5 \mathrm{~mm}$, Lower Left) $Z=50 \mathrm{~mm}$, Upper Right) $\mathrm{Z}=100 \mathrm{~mm}$ and Lower Right) $\mathrm{Z}=300 \mathrm{~mm}$.

Figure 3 shows the radial profiles of axial velocity for Swirl numbers $0.14,0.64$ and 0.74 in different cross sections located at $Z=5,25,50$ and $100 \mathrm{~mm}$ from the discharge of the nozzles as well as the experimental results from Palm et al. [10]. These experimental results are for a swirl of 0.6. Every section evidences the increase of momentum mixing while increasing the swirl. The sections $Z=5 \mathrm{~mm}, 25$ and $50 \mathrm{~mm}$ show that the numerical results are less dissipative than experimental results but section $Z=100 \mathrm{~mm}$ section shows a good agreement with the experimental results. Negative values of the axial velocity evidence the existence of recirculation zones. It is clear the presence of ORZ in sections 25 and $50 \mathrm{~mm}$ for any swirl. As for the IRZ, it only appears for Swirl 0.74 .

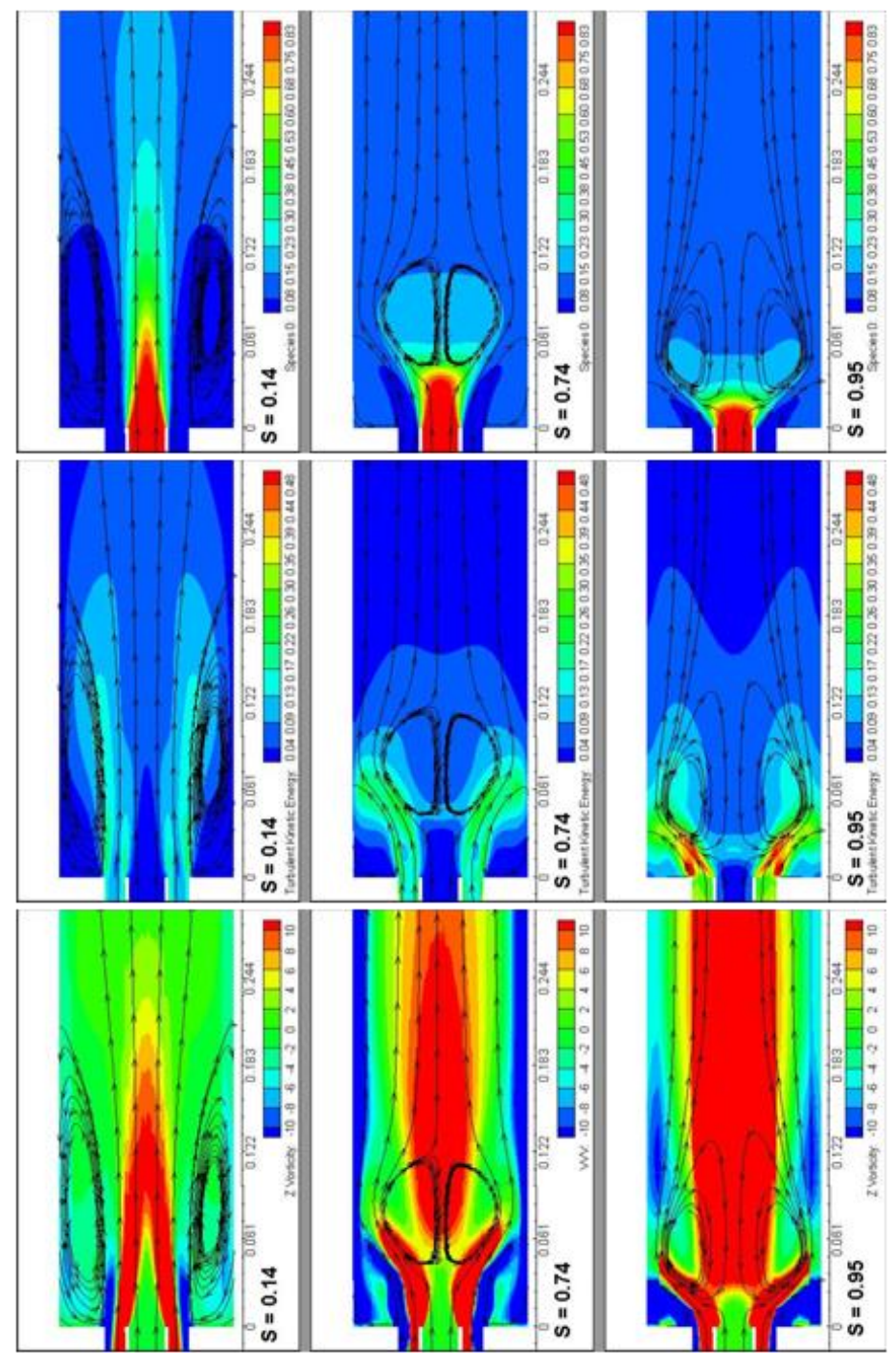

Fig. 5. Contours in the plane $x=0$ and stream lines to locate recirculation zones. Top) Passive scalar, Centre) Turbulent Kinetic Energy and Bottom) Axial Vorticity for different Swirl numbers Left) $S=0.14$, Middle) $S=0.74$, Right) $S=0.95$.

Figure 4 shows the radial profiles of temperature for Swirl numbers 0.14 and 0.74 in different cross sections located at $Z=5,50,100$ and $300 \mathrm{~mm}$ from the discharge of the nozzles. Every section affected by the IRZ, Z > $5 \mathrm{~mm}$ and Swirl >0.6, evidences the increase of 
heat transfer while increasing the swirl. Mass fraction shows similar results in the same sections. It is clear the homogenization role played by the IRZ for high swirl numbers.

Figure 5 show longitudinal contours of the passive scalar, turbulent kinetic energy and axial vorticity. The first one let identify mass mixing. Steam lines let identify the IRZ concluding the important role played in homogenizing species. Turbulent kinetic energy show maximum values on the shear layer but there is not relevant production of energy inside the IRZ. Finally, the axial vorticity is located on the annular jet because of the swirler and in the IRZ, where fluid spin as a rigid solid, with minimum deformation.

\section{Conclusion}

This paper studies the mixing of different swirling jets ranging from low to intermediate and high swirling numbers. Validation was provided for high swirl number.

Low swirling injectors does not promote the fluid to turn over near the centre of the chamber, resulting larger mixing zones with weak gradients. Pressure distribution for intermediate and larger swirls promote the formation of a vortex bulb near the axis of the chamber. This inner recirculation zone is responsible of deflecting the shear layer and increases the gradients near the upstream of its lead stagnation point. As a result, mixing occurs at short distance of the nozzle's discharge. Therefore it is justified the common use of swirling to burn lean mixtures that would show instable behaviour without any swirl.

Also the convex or concave shape of the recirculation point near this stagnation point is significant on the mass and heat transfer. The former appears for Swirl number 0.74 whereas the latest appears for Swirl $=0.95$. Future works involve the study of reactive cases.

\section{Acknowledgements}

The authors thankfully acknowledge the Spanish Ministry of Science and Innovation for the financial resources in the framework of the project reference ENE2011-25468.

We acknowledge PRACE for awarding us access to resource Curie-Genci based in France at CEA and MareNostrum based in Spain at BSC. Ref. 2010PA1766.

\section{References}

[1] T. Parra, R. Z. Szasz, C. Duwig, R. Pérez, V. Mendoza and F. Castro, "Acoustic Instabilities on Swirling Flames," International Journal of Mechanical Engineering, vol. 7, no. 9, pp. 742-745, 2013.

[2] H. S. Zhen, C. S. Cheung, C.W. Leung and H.B. Li, "Thermal and heat transfer behaviors of an inverse diffusion flame with induced swirl," Fuel, vol. 103, pp. 212-219, 2013.

[3] J. Guo, Y. Yan, W. Liu, F. Jiang and A. Fan, "Enhancement of laminar convective heat transfer relying on excitation of transverse secondary swirl flow," International Journal of Thermal Sciences, vol. 87, pp. 199-206, 2015.

[4] K. M. Saqr and M. A. Wahid, "Effects of swirl intensity on heat transfer and entropy generation in turbulent decaying swirl flow," Applied Thermal Engineering, vol. 70, pp. 486-493, 2014.

[5] H. Gül and D. Evin, "Heat transfer enhancement in circular tubes using helical swirl generator insert at the entrance," International Journal of Thermal Sciences, vol. 46, pp. 1297-1303, 2007.

[6] C. Nuntadusit, M. Wae-hayee, A. Bunyajitradulya and S. Eiamsa-ard, "Heat transfer enhancement by multiple swirling impinging jets with twisted-tape swirl generators," International Communications in Heat and Mass Transfer, vol. 39, pp. 102-107, 2012.

[7] A.E. Zohir and A.G. Gomaa, "Heat transfer enhancement through sudden expansion pipe airflow using swirl generator with different angles," Experimental Thermal and Fluid Science, vol. 45, pp. 146-154, 2013.

[8] A.E. Zohir, A.A. Abdel Aziz and M.A. Habib, "Heat transfer characteristics in a sudden expansion pipe equipped with swirl generators," International Journal of Heat and Fluid Flow, vol. 32, pp. 352-361, 2011.

[9] R. Roback and B.V. Johnson, "Mass and momentum turbulent transport experiments with confined swirling coaxial jets," Interim Report, 1983.

[10] R. Palm, S. Grundmann, M. Weismuller, S. Saric, S. Jakirlic and C. Tropea, "Experimental characterization and modelling of inflow conditions for a gas turbine swirl combustor," International Journal of Heat and Fluid Flow, vol. 27, pp. 924-936, 2006.

[11] T. Parra-Santos, J. R. Perez-Dominguez, R. Z. Szasz and F. Castro, Engineering Computations. Accepted 23.07.2014.

[12] T. Parra-Santos, J. R. Perez-Dominguez, R.Z. Szasz and F. Castro, "An isothermal analysis of curved-vane 
and flat-vane swirlers for burners," Engineering Computations, vol. 32, no. 3, pp. 668-686, 2015.

[13] J. H. Ferziger and M. Peric, Computational Methods for Fluid Dynamics, $3^{\text {rd }}$ edition, Springer, 2002.

[14] H. K. Versteeg and W. Malalasekera, An Introduction to Computational Fluid Dynamics, The Finite Volume Method, Longman Group Ltd, 1995.

[15] T. Parra, V. Vuorinen, R. Perez, R. Szasz and F. Castro, "Aerodynamic characterization of isothermal swirling flows in combustors," International Journal of Energy and Environmental Engineering, vol. 5, no. 2, pp. 1-6, 2014.

[16] V. Yakhot, S. A. Orszag, S. Thangam, T. B. Gatski and C. G. Speziale, "Development of turbulence models for shear flows by a double expansion technique," Physics of Fluids A, vol.4, no. 7, pp. 1510-1520, 1992.

[17] M.García-Villalba and J.Fröhlich, "LES of a free annular swirling jet-Dependence of coherent structures on a pilot jet and the level of swirl," International journal of heat and fluid flow, vol. 27, no. 5, pp. 911-923, 2006.

[18] T. Parra, R. Perez, G. Lorenzo, R. Szasz, A. Gutkowski and F. Castro, "Diffuser influence on the mixing of coaxial swirling jets," ASME FEDSM201421354, 2014. 